\title{
Empathic assistants - Methods and use cases in automated and non-automated driving
}

Anna-Antonia Pape ${ }^{1}$, Sonja Cornelsen ${ }^{1}$, Victor Faeßler ${ }^{1}$, Klas Ihme ${ }^{2}$, Michael Oehl ${ }^{2}$, Uwe Drewitz ${ }^{2}$, Franziska Hartwich ${ }^{3}$, Frank Schrödel ${ }^{4}$, Andreas Lüdtke ${ }^{5}$, Martin Schramm ${ }^{6}$

1: TWT GmbH Science \& Innovation

2: Deutsches Zentrum für Luft- und Raumfahrt, Braunschweig

3: Technische Universität Chemnitz

4: IAV Automotive Engineering, Chemnitz

5: OFFIS Institut für Informatik, Oldenburg

6: Soundreply GmbH, Köln 


\section{Introduction}

Modern vehicles come with various advanced driving assistance systems. Today, most of these systems are targeted at improving safety, because human error is a major cause for accidents. Most human errors are related to impaired cognitive processing due to sleepiness, alcohol intoxication or engagement in secondary tasks such as cell phone use that distract from the driving task. Therefore, systems that monitor driver states for improved safety are on the market and will be mandatory in new cars starting in 2022 $[1]$.

However, with the advent of self-driving cars, human driving errors are deleted from the equation. This means that safety becomes less of an issue because the human is no longer the driver. Instead, tomorrow's passenger will want to use the free time relaxing, working or doing something fun [2] and, he ${ }^{1}$ will want to switch between these activities quickly without too much hassle. That means with the arrival of self-driving cars, new needs and expectations have to be catered to both with hardware, e.g. for working or sleeping, but also with intuitive human machine interfaces to operate that hardware.

Despite its many promises, many people have concerns about autonomous cars according to previous research [3]. They therefore plan to either not use them at all or plan to keep watching the road, thereby missing out on the benefits autonomous vehicles have to offer to their users. These challenges, if not addressed, will at best severely impair the user experience in self-driving cars and at worst reduce the acceptance of this technology in a way that its market spread is delayed.

To help tomorrow's passenger enjoy the ride, we suggest to repurpose the technology for use in empathic assistants [4,5,6,7]. An in-car empathic assistant is a digital assistant whose goal is to make driving comfortable and safe by catering to the user's needs. To this end, it detects user states and tries to improve the situation either by directly executing or by suggesting in-car adaptations tailored to the user need in the situation.

Such interventions can be anything that improves the comfort of the passenger. If the passenger is sleepy, the empathic assistant could carefully recline the chair and make sure that driving maneuvers are gentle and don't wake the passenger up. If the passenger wants to work, the empathic assistant could reduce the number of notifications or block incoming calls and choose route alternatives where working is easy (e.g. less bends and curves).

\footnotetext{
${ }^{1}$ Whenever the personal pronoun „he“ is used, it should be understood as referring to all genders, unless explicitly stated.
} 
As we will see, use cases of empathic assistants are not limited to automated driving. In fact, for manual driving contexts, a pro-active human co-driver is the role model of an empathic assistant. Just like a pro-active caring human co-driver understands the current situation inside and outside the car, the empathic assistant understands the driver and takes care that the atmosphere in the car is as comfortable as possible and actively supports the driver by suggesting and/ or completing small secondary tasks. An example of pro-active support could be to turn the radio sound lower on a busy intersection if the co-driver notices signs of increased workload in the driver or knows the driver to be sensitive to noise in stressful situations.

In our work on empathic assistants, we put the user and his needs into the center of our attention and develop for his needs in the car. In the remainder of the paper, we will shortly talk about the technical building blocks of empathic assistants before describing our work on two empathic assistants we are currently developing in two federallyfunded research projects, namely AutoAkzept and F-RELACS.

\section{Technical building blocks of empathic assistants}

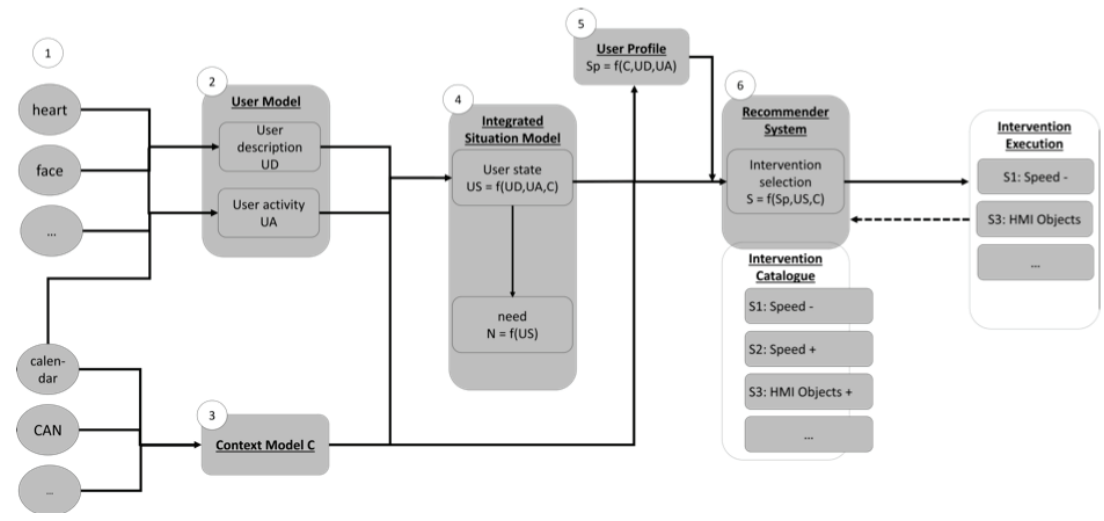

Figure 1: System Architecture of an Empathic Assistant, see [8]

To implement an empathic assistant as a helpful digital companion in cars, a comprehensive system architecture is necessary, see Figure 1. We designed and currently implement this architecture in AutoAkzept [8]. It is implemented in a similar way in 
F-RELACS. The architecture consists of six modules. On one end of the system architecture is a set of sensors (1) that monitor the user ${ }^{2}$ and the context and uses them to model both user and context separately $(2,3)$. Information about the user and the context is then integrated in the integrated situation model (4). A user profile (5) holds information about user preferences. In the end, a recommender system (6) suggests the best possible reaction or action that the empathic assistant can take.

Data about the user is continuously captured using contactless sensors such as cameras tracking his face, eyes and body, and sensors monitoring e.g. heart rate and skin conductance via electrodes or photoplesmography (PPG). These data are fed into machine learning classifiers that have been trained to detect certain behaviors ("e.g. taps with his foot", "bites his lips") and categorized physiological states ("has increased heart rate").

To monitor the context, the system taps into several different environmental data that may have an influence on the user's current state. These data can include recent interactions with the car HMI, calendar entries on the user's phone, the time of day, the car's current speed, its time-headway, etc. The context model (3) clusters the environmental data into categories that have been found to correlate with certain user states.

The integrated situation model (4) integrates information from the user model and the context model and derives the most likely user state along with its most likely environmental cause (e.g. P(anxious| time pressure)) using Bayesian methods.

Module (5) and (6) have slightly similar purposes: they suggest the best intervention strategy that the empathic assistant should take. The user profile (5) outputs an intervention strategy that a specific user would most likely prefer given his personal history of previous interventions and his reactions to them [9].

As this user profile is not always available or the preferred intervention may not be suitable, the recommender system (6) after additional security checks makes the final decision. It chooses the most suitable intervention strategy to improve the current user experience and transfers it for execution.

\footnotetext{
${ }^{2}$ For ease of reading, we will use the word "user" to refer to the human whose cognitive or emotional state is being inferred. In a manual driving situation, the user is the driver, in an autonomous driving situation any passenger of the car can be the user.
} 


\title{
AutoAkzept - An empathic agent improving trust in automated vehicles
}

AutoAkzept puts the human passenger and his needs during automated and autonomous driving into the center of our research and development. In this project, we are developing solutions for three uses cases, namely (1) enabling working in the mobile office, possibly under time pressure, (2) improving trust and comfort in a RoboTaxi, and (3) preventing kinetosis [8].

Regarding use case 2, previous research has shown that passengers often feel discomfort and anxiety when riding in autonomous cars because maneuver decisions may seem unsafe from the perspective of the human passenger [10]. This occurs for example when the car keeps small distances to leading cars or overtakes when the human driver perceives it to be unsafe. However, under the assumption that the automation makes sound decisions (e.g. because it is wirelessly connected to the leading car and shares information about maneuvers with it), the passengers' feelings of discomfort and anxiety are unnecessary and can and should be avoided through an empathic assistant.

The goal of our work in AutoAkzept is a demonstration of an empathic assistant than can address the user needs in all three use cases. Here, we present parts of this work addressing the user story in the scenario named Shared Space which is part of the use case nr 2 (RoboTaxi):

\section{User Story Shared Space Scenario in the UseCase RoboTaxi in AutoAkzept}

\begin{abstract}
It is Saturday. You just finished shopping downtown and it's very busy and crowded. With all those heavy, voluminous bags you don't want to block the public transport. Luckily, as you leave the store, you spot a self-driving taxi and grab it. As you sink relieved into the seat, the taxi leaves the parking spot and everything seems fine, but then your way leads across a shared space. Your relief is exchanged by anxiety for all the pedestrians and bikes around the taxi. Has the car seen that bike next to us and that it seems to want to go straight where the taxi needs to turn right? And what about the mother with her little girl wanting to cross our path...?
\end{abstract}

In the user story above, the empathic assistant should take measures to relieve the passenger from his anxiety, e.g. by sharing information about the car's situational model of the surroundings or upcoming trajectory. This should be done when it's necessary, i.e. when he is anxious about how the car handles the traffic. It should not be done at uninvited or unnecessary times to avoid annoying the user with unwanted information, e.g. when the user is sleeping or working. 
That means, to put the "empathic" into the empathic assistant, the assistant needs to be able to know when to assist. This is why we need user state detection, i.e. machine learning algorithms that have been trained to differentiate between anxious and nonanxious users and can infer from the context whether present anxiety is caused by the driving situation.

To train the algorithms, large amounts of data are necessary that have to be captured from real users in contexts that match the real application as closely as possible. We collected data from 17 participants riding on the passenger seat of a self-driving car through seven everyday scenes with several pedestrians and a bike (see Figure 2). For reasons of safety and experimental design, the experiment was carried out on the runway of a small airport. All participants (9 women) had a driver's license and drove at least $3.000 \mathrm{~km} /$ year. Mean age was 28.88 (range $=20-50)$.

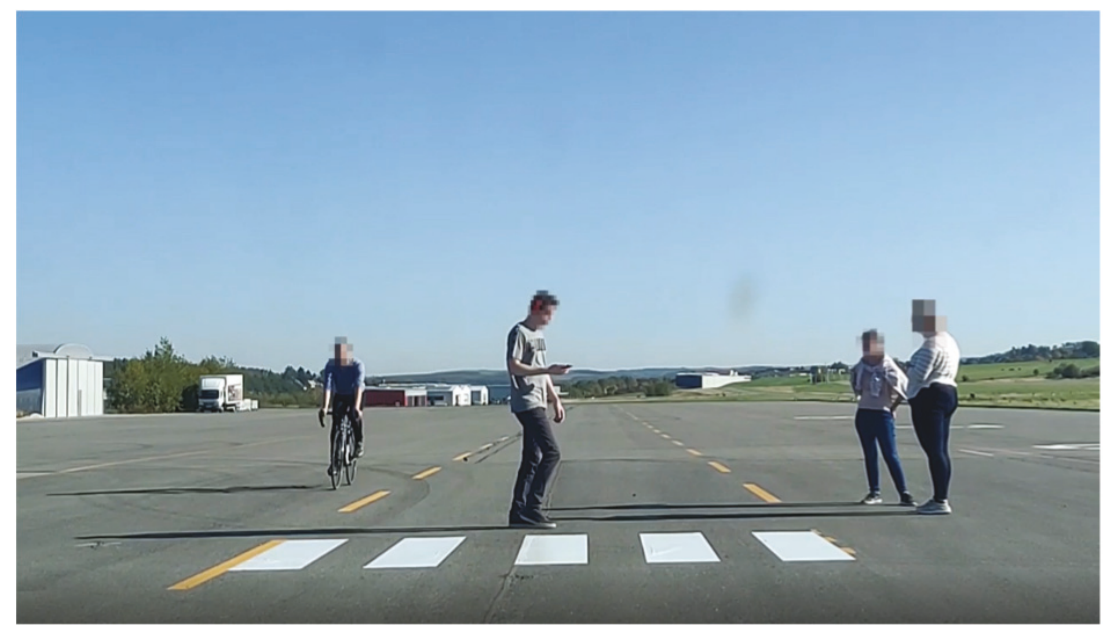

Figure 2: Scene in which a pedestrian crosses the car's path while he is wearing head-phones and staring at the phone. Anxiety could arise because the pedestrian remains unaware of the approaching self-driving car and if the car did not start breaking early enough for the passenger to feel safe.

The data we collected from the participants during this experiment included camera data observing the participant's face and body, eyetracker-data, and data on physiological variables (heart rate and skin conductance) collected by a wristband worn on the left arm. In addition, participants continuously rated their anxiety level using a tracking device to be operated with their right hand's index finger. 
The data collected for context observation included information from the car's environmental sensors, e.g. time headway, position and path of detected objects in the car's vicinity and so on.

After each scene, participants rated the potential sources of anxiety, e.g. whether they felt the car was able to handle the situation, whether they felt well informed about the upcoming behavior of the car, whether it kept enough distance to the pedestrian and to the bike and so on. Based on these data, we are currently developing user models and an integrated situation model to link user states and likely causes.
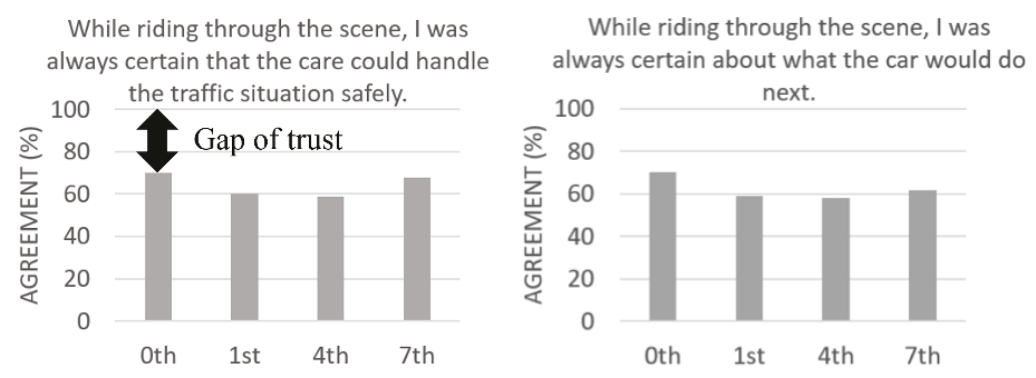

Figure 3: Average agreement $(\mathrm{N}=17)$ to two statements after riding on the passenger seat of an automated car through different scenes without and with other road users. In the 0th ride, no other road users were present. The 1 st, 4 th and 7 th scene participants experienced different everyday scenes with pedestrians and bikes.

In addition, the results from these questionnaires (Figure 3, left panel) are in line with the rationale of the project, because they show that participants reported a gap of trust in the abilities of the self-driving car. Also, this gap slightly increased, at least initially, once other road user were present on scene. At the end of the experiment, after experiencing seven scenes, the trust gap still remained.

In a similar way, participants felt they could not foresee what the car would be doing next (Figure 3, right panel). To find out whether information about the egocar's upcoming behavior would be helpful, we asked after each scene which information they would have liked to have while riding in the car. And indeed, most participants agreed that information about the intention of the egocar (i.e. the car in which participants were sitting) would be very helpful. Information about the position and inferred information about the intention of the other road users was also rated to be helpful with slightly less between-subject agreement (Figure 4).

The questionnaires gave us a first insight in what kind of information should be offered by the empathic assistant to improve user experience. To get a more detailed insight 
into our user's thoughts, we also conducted an interview with each participant after he experienced all seven scenes. In the later part of this interview we used a rapid prototyping method to iteratively improve prototypes on human machine interfaces to communicate helpful information to allow the user to better understand the maneuver decisions and reduce anxiety. Based on the interviews, we are currently developing a headdown and a head-up HMI using our driving simulators and virtual reality set-ups.

As mentioned before, this paper describes just one of three scenarios in one of three use cases that we're currently building demonstrators for in AutoAkzept. Together, our developments on empathic assistants will contribute to better user experience and ultimately acceptance of self-driving cars.

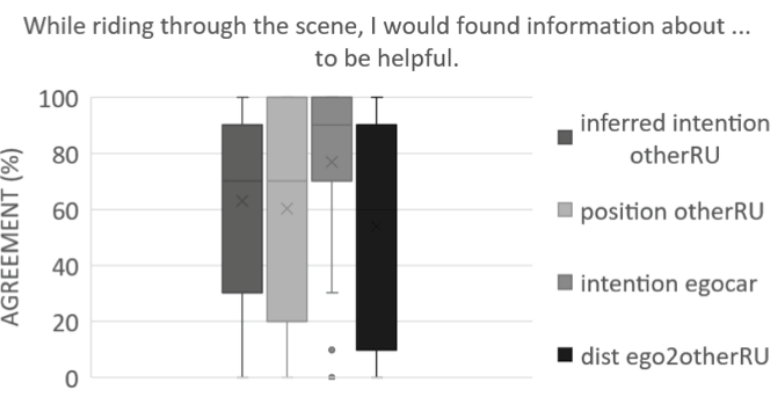

Figure 4: Average agreement $(\mathrm{N}=17)$ to statements about which information participants deemed helpful. Abbreviated axis labels refer to the information about (left to right) intention of other road users inferred by the car, position of the other road users, intention of the egocar (the car in which participants were riding) and distance between the egocar and the other road users.

\section{F-RELACS - An empathic assistant reducing frustration in two automation levels}

Negative emotions such as frustration are common in traffic and while driving. When drivers experience negative emotions such as frustration, they are most often triggered by events happening in the surrounding traffic or while interacting with the navigation system or other parts of the human computer interface [11]. For example, triggers of frustration can be congested roads, slow leading vehicles or red traffic lights that hinder the driver from reaching his destination at the expected time $[12,13,14]$. These examples demonstrate that frustration can occur in all automation levels. Ergo, many possible use cases can be thought of where an empathic assistant may sense and reduce frustration. 
Also, frustration has practical implications for safety and user experience, underlining the value of a frustration-reducing empathic assistant. Not only is frustration generally unpleasant and but it is also detrimental to user experience, if it is directed at the interaction between user and a certain technology and will limit acceptance and spread of this technology. In addition, frustration can also turn into a safety issue: Previous research has shown that frustration and anger can lead to aggressive driving and more generally impairs cognitive processing ability needed for driving [4,12].

Having identified frustration as a worthwhile challenge and playfield for an empathic assistant because it impairs both safety and user experience, in the federally-funded research project F-RELACS (Frustration Real-Time Recognition for an Adaptive InCar System) we work on developing an empathic assistant that reduces in-car frustration.

First, in a workshop with experts from automotive human factors, human-machine interaction and voice user interfaces, we generated user stories describing concrete frustrating scenarios, and picked one from the field of manual driving, one from the field of automated driving. These user stories are the basis of all our work in F-RELACS.

\section{User Stories in F-RELACS}

\begin{tabular}{|c|c|}
\hline $\begin{array}{l}01 \text { Autonomous Driving / User Experi- } \\
\text { ence }\end{array}$ & 02 Manual Driving / Safety \\
\hline $\begin{array}{l}\text { Paul is going to an important business } \\
\text { meeting in another city in a rented, auton- } \\
\text { omous car. Halfway there, he receives a } \\
\text { call from the day care of his child, that his } \\
\text { son needs to be picked up immediately. } \\
\text { Because he has no other option, Paul de- } \\
\text { cides to go pick up his son, but first he } \\
\text { has to manage to reprogram the naviga- } \\
\text { tion system. Because the navigation sys- } \\
\text { tem is confusing, this turns out to be quite } \\
\text { a hassle and while the car keeps going to- } \\
\text { wards the business meeting, Paul be- } \\
\text { comes more and more frustrated. }\end{array}$ & $\begin{array}{l}\text { Tina wants to meet her friends in the } \\
\text { neighbor town to go see a movie with } \\
\text { them. On the rural streets that lead her } \\
\text { there, she gets stuck behind a slow } \\
\text { truck and cannot overtake due to heavy } \\
\text { traffic on the opposite lane. As she } \\
\text { gets a message from her friends that } \\
\text { the parking options are more limited } \\
\text { than usual, her frustration level rises. } \\
\text { When she finally enters the city, she } \\
\text { needs to take a left turn, but the green } \\
\text { light phases are painfully short delay- } \\
\text { ing her even further. }\end{array}$ \\
\hline
\end{tabular}

To find out what users perceive as helpful strategies to reduce frustration, we conducted a focus group study. Here, one often-mentioned strategy was a voice user interface (VUI) that assists the user in various ways through natural speech [15]. 
Indeed, use of in-car voice user interfaces is wide-spread [16]. There are more monthly users of in-car voice user interfaces than monthly users of smart home speakers. Also, previous results suggest that empathic voice user interfaces seem to work in reducing anger or sadness [17]. Based on these promising previous results and wide-spread use of in-car voice user interfaces, we decided to investigate the questions whether people accept voice user interfaces for reduction of frustration and the specific variables that make an empathic voice assistant effective for reduction of frustration.

To reduce frustration using digital assistants, several paths can be taken [18]: Of course, removing the cause of frustration would immediately resolve frustration. However, this is not always possible as in the case of slow leading traffic or traffic jams and construction sites (user story 2). Therefore, we are currently exploring two voice-based interventions strategies that may reduce frustration even if the immediate cause cannot be removed.

On the one hand, we assess whether user's frustration is reduced when the voice user interface employs emotion regulation strategies. Theoretically, this work in grounded on Gross' process model of emotion regulation [19]. Specifically, we test whether and how the success of the intervention depends on timing [20].

Another strategy we explore is to manipulate the VUI's prosody, i.e. the tone of voice. Prosody is an important element of natural conversation. Not only does it often help the listener to recognize the speaker's emotion and intention, it can also be an important display of affective empathy, the capacity to respond with an appropriate emotion to another's mental state. In therapy, changing just the prosody of a statement, can make the difference in the effect of a therapist's response to his client, either validating or challenging the client [21]. Thus, we want to test the effect of natural prosody in in-car VUI and whether it is helpful to reduce frustration. Indeed, an earlier study showed that matching a VUI's prosody to the driver's mood, subjectively and objectively improved attention measures and led to less driving mistakes [22]. In our experiment we test whether participant's frustration is reduced by comments and support offered by an understanding voice aimed to validate the feeling of frustration. 


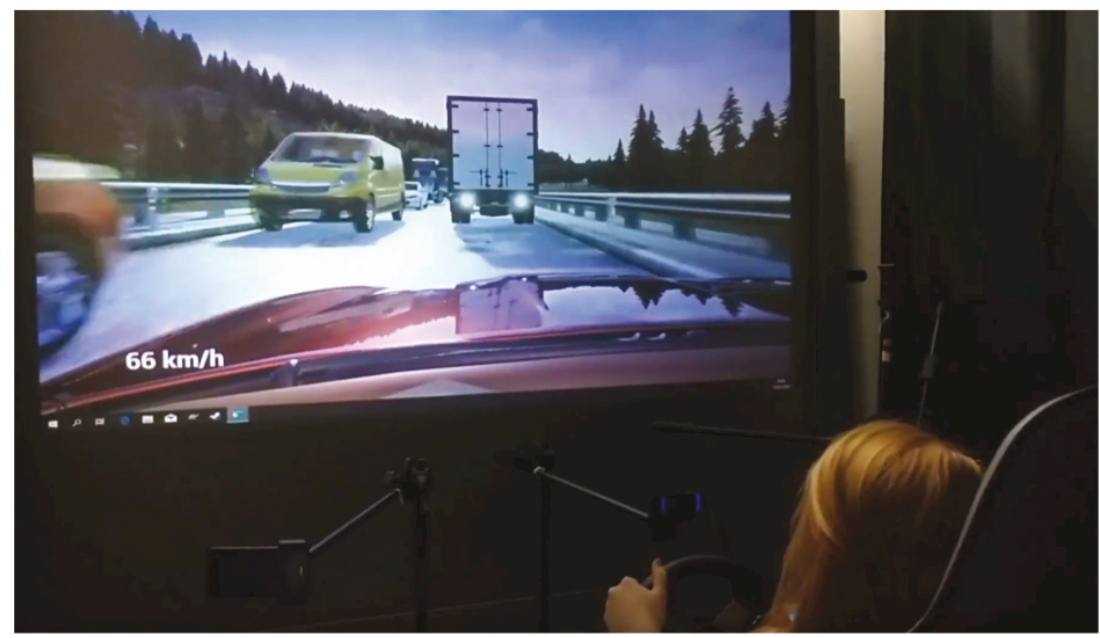

Figure 5: The picture shows part of our simulator set-up used to manipulate and record user states. The screen shows a frustrating scene in F-RELACS use case 02 (frustration in manual driving) built in our product Tronis ${ }^{\circledR}$.

In parallel to our work on frustration intervention strategies, we are currently also collecting data on user's expression of frustration (see Figure 5). As of now, the corpus already contains data of 50 participants. The experiments are carried out partly in our driving simulators and partly in an automated car. While we use a cover story to distract from the real purpose of the study, we do manipulate the participants' frustration level. This allows us to collect expressions of frustration as they naturally occur using cameras, eyetracking and physiological sensors. The data is used to train machine learning algorithms that can detect different levels of frustration to allow the empathic voice assistant of F-RELACS to jump in and be helpful when needed by responding with appropriate, suitable frustration mitigation strategies.

\section{Conclusion and Outlook}

We presented our ongoing work on developing empathic assistants for automated and non-automated driving.

Empathic assistants can be useful for both safety and comfort applications because they sense user states, their likely environmental causes and infer the user's needs and suitable adaptations or interventions to these needs. 
When developing empathic assistants, two aspects are especially important: The adaptations and interventions need to be as situation-specific and solution-oriented as possible because general unspecific strategies have shown to be less effective on changing user states [17]. Second, the interventions and suggestions need to come at the right time. We address these aspects with the comprehensive system architecture presented.

Of course, there are limits to this approach. For example, as of right now it is unclear how to react to user states that are the result of situations that have happened either in the past or are generally not related to the current situation. In such situations, the empathic assistant will not be able to find the cause of the user state and therefore cannot react with situation-specific and solution-oriented adaptations. How to handle this situation in the best manner needs to be dealt with to maintain a high user acceptance and trust towards the empathic assistant itself.

Another current limitation is how to address individual differences and preferences about different interventions and adaptations. While we do develop a user profile module to keep track of individual user preferences, this user profile initially needs to learn the preferences and may need thousands of data points on each individual user to be able to do that reliably. Therefore, during the learning phase, the adaptations may not match the individual's preferences but an adaptation that matches the taste of most users.

Last but not least, it may be worthwhile to broaden the applications of empathic assistants beyond improving safety and comfort by reacting to negative user states towards empathic assistants that strengthen and foster already present positive user states such as happiness [23].

\section{Acknowledgments}

AutoAkzept is funded by the German Federal Ministry of Transport and Digital Infrastructure (BMVi) (grant number: 16AVF2126). The consortium is led by the German Aerospace Center (DLR) in Braunschweig. The consortium further consists of the Chemnitz University of Technology, IAV GmbH in Chemnitz, TWT GmbH Science \& Innovation in Stuttgart, and OFFIS in Oldenburg.

F-RELACS is funded by the German Federal Ministry of Education and Research (grant number: 16SV7929K). The consortium is led by TWT GmbH Science \& Innovation in Stuttgart, and further consists of the German Aerospace Center (DLR) in Braunschweig and Soundreply GmbH in Köln. 
Empathic assistants - Methods and use cases in automated and non-automated ...

\section{Bibliography}

1. Road safety: Commission welcomes agreement on new EU rules to help save lives. (2019). Retrieved January 20, 2020, from https://ec.europa.eu/commission/ presscorner/detail/en/IP_19_1793

2. Audi is researching the use of time in the robot car. (2017). Retrieved October 11, 2019, from https://www.audi-mediacenter.com/en/press-releases/audi-is-researching-the-use-of-time-in-the-robot-car-9120

3. Schoettle, B. S., \& Sivak, M. (2014). A survey of public opinion about autonomous and self-driving vehicles in the U.S., the U.K., and Australia. Retrieved January 20, 2020, from https://deepblue.lib.umich.edu/handle/2027.42/108384

4. Jeon, M. (2015). Towards affect-integrated driving behaviour research. Theoretical Issues in Ergonomics Science, 16(6), 553-585. https://doi.org/10.1080/ $1463922 x .2015 .1067934$

5. Large, D. R., Burnett, G., \& Clark, L. (2019). Lessons from Oz. Proceedings of the 11th International Conference on Automotive User Interfaces and Interactive Vehicular Applications Adjunct Proceedings - AutomotiveUI '19. https://doi.org/ $10.1145 / 3349263.3351314$

6. Vögel, H.-J., Troncy, R., Huet, B., Önen, M., Ksentini, A., Conradt, J., ... Härri, J. (2018). Emotion-awareness for intelligent vehicle assistants. Proceedings of the 1st International Workshop on Software Engineering for AI in Autonomous Systems SEFAIS '18. https://doi.org/10.1145/3194085.3194094

7. Stephanidis, C., Salvendy, G., Antona, M., Chen, J. Y. C., Dong, J., Duffy, V. G., ... Zhou, J. (2019). Seven HCI Grand Challenges. International Journal of Human-Computer Interaction, 35(14), 1229-1269. https://doi.org/10.1080/ 10447318.2019.1619259

8. Drewitz, U., Ihme, K., Oehl, M., Schrödel, F., Voßwinkel, R., Hartwich, F., Schmidt, C., Pape, A.-A., Fleischer, T., Cornelsen, S., Lüdtke, A., Gräfing, D., \& Trende, A. (2019). Automation ohne Unsicherheit: Vorstellung des Förderprojekts AutoAkzept zur Erhöhung der Akzeptanz automatisierten Fahrens. In VDI (Ed.). Mensch-Maschine-Mobilität 2019. Der (Mit-)Fahrer im 21. Jahrhundert!? VDI-Berichte 2360 (pp. 1-19). Düsseldorf: VDI Verlag. ISBN: 978-3-18-092360-4

9. Trende, A., Gräfing, D., \& Weber, L. (2019). Personalized user profiles for autonomous vehicles. Proceedings of the 11th International Conference on Automotive User Interfaces and Interactive Vehicular Applications: Adjunct Proceedings - AutomotiveUI '19, 287-291. https://doi.org/10.1145/3349263.3351315 
10.Beggiato, M., Hartwich, F., \& Krems, J. (2018). Using Smartbands, Pupillometry and Body Motion to Detect Discomfort in Automated Driving. Frontiers in Human Neuroscience, 12. https://doi.org/10.3389/fnhum.2018.00338

11.Zepf, S., Dittrich, M., Hernandez, J., \& Schmitt, A. (2019). Towards Empathetic Car Interfaces. Extended Abstracts of the 2019 CHI Conference on Human Factors in Computing Systems - CHI EA '19, LBW0129, 1-6. https://doi.org/ $10.1145 / 3290607.3312883$

12.Lee, Y.-C. (2010). Measuring Drivers' Frustration in a Driving Simulator. Proceedings of the Human Factors and Ergonomics Society Annual Meeting, 54(19), 15311535. https://doi.org/10.1177/154193121005401937

13.Ihme, K., Dömeland, C., Freese, M., \& Jipp, M. (2018). Frustration in the face of the driver. Interaction Studies, 19(3), 487-498. https://doi.org/10.1075/is.17005.ihm

14.Ihme, K., Unni, A., Zhang, M., Rieger, J. W., \& Jipp, M. (2018). Recognizing Frustration of Drivers From Face Video Recordings and Brain Activation Measurements With Functional Near-Infrared Spectroscopy. Frontiers in Human Neuroscience, 12(327). https://doi.org/10.3389/fnhum.2018.00327

15.Oehl, M, Ihme, K., Pape, A., Vukelic, M., \& Braun, M. (2020). Affective Use Cases for Empathic Vehicles in Highly Automated Driving: Results of an Expert Workshop. HCII 2020: Proceedings of the 22nd International Conference on Human Computer Interaction. Copenhagen, Denmark, 19-24.

16. Kinsella, B. (2019). In-Car Voice Assistant Consumer Adoption Report (January). Retrieved from https://voicebot.ai/wp-content/uploads/2019/01/in-car_voice_assistant_consumer_adoption_report_2019_voicebot.pdf

17.Braun, M., Schubert, J., Pfleging, B., \& Alt, F. (2019). Improving Driver Emotions with Affective Strategies. Multimodal Technologies and Interaction, 3(1), 21. https://doi.org/10.3390/mti3010021

18. Hone, K. (2006). Empathic agents to reduce user frustration: The effects of varying agent characteristics. Interacting with Computers, 18(2), 227-245. https://doi.org/10.1016/j.intcom.2005.05.003

19. Gross, J. J. (1998). The emerging field of emotion regulation: an integrative review. Review of General Psychology, 2(3), 271-99.

20.Lienhop, M., \& Oehl, M. (2020). Taking driver frustration into account: Towards a speech-based emotion regulation in-car assistant. Abstract accepted at TeaP 2020, Jena 
21.Weiste, E., \& Peräkylä, A. (2014). Prosody and empathic communication in psychotherapy interaction. Psychotherapy Research, 24(6), 687-701. https://doi.org/10.1080/10503307.2013.879619

22.Nass, C., Jonsson, I.-M., Harris, H., Reaves, B., Endo, J., Brave, S., \& Takayama, L. (2005). Improving automotive safety by pairing driver emotion and car voice emotion. CHI '05 Extended Abstracts on Human Factors in Computing Systems CHI '05, 1973-1976. https://doi.org/10.1145/1056808.1057070

23.Oehl, M, Ihme, K., Pape, A., Vukelic, M., \& Braun, M. (2020). Affective Use Cases for Empathic Vehicles in Highly Automated Driving: Results of an Expert Workshop. HCII 2020: Proceedings of the 22nd International Conference on Human Computer Interaction. Copenhagen, Denmark, 19-24. 